\title{
Criminologie
}

\section{L'homicide conjugal féminin, de Marie-Josephte Corriveau (1763) à Angélique Lyn Lavallée (1990) : meurtre ou légitime défense ?}

\section{Sylvie Frigon}

Volume 29, numéro 2, automne 1996

L'homicide conjugal au Canada

URI : https://id.erudit.org/iderudit/017387ar

DOI : https://doi.org/10.7202/017387ar

Aller au sommaire du numéro

Éditeur(s)

Les Presses de l'Université de Montréal

ISSN

0316-0041 (imprimé)

1492-1367 (numérique)

Découvrir la revue

Citer cet article

Frigon, S. (1996). L'homicide conjugal féminin, de Marie-Josephte Corriveau (1763) à Angélique Lyn Lavallée (1990) : meurtre ou légitime défense ? Criminologie, 29(2), 11-27. https://doi.org/10.7202/017387ar
Résumé de l'article

In this article, the author examines the socio-legal relevance of the " battered woman syndrome " (BWS) in the cases of women who kill their violent partners in Canada. The legal recognition of domestic violence in these situations, in its historical context, is examined by focusing on the Supreme Court of Canada decision in the case of Angélique Lyn Lavallée in 1990. This landmark decison is used as pivotal in this discussion in order to shed light into the reasoning behind the subsequent legal decisions. The ideas proposed in this article are part of a more general exploration of the representations of femininity and their inscription in law. In discussing female conjugal homicide, the gendered nature of law and order, the pathologization of women, the "syndromisation" phenomenon and the médicalisation of violence, the author offers some insights in thinking legal strategies but more fundamentally in the social debate to continue. 


\section{L'HOMICIDE CONJUGAL FÉMININ DE MARIE-JOSEPHTE CORRIVEAU (1763) À ANGÉLIQUE LYN LAVALLÉE (1990) : MEURTRE OU LÉGITIME DÉFENSE ? \\ Sylvie Frigon'}

In this article, the author examines the socio-legal relevance of the "battered woman syndrome" (BWS) in the cases of women who kill their violent partners in Canada. The legal recognition of domestic violence in these situations, in its historical context, is examined by focusing on the Supreme Court of Canada decision in the case of Angélique Lyn Lavallée in 1990. This landmark decison is used as pivotal in this discussion in order to shed light into the reasoning behind the subsequent legal decisions. The ideas proposed in this article are part of a more general exploration of the representations of femininity and their inscription in law. In discussing female conjugal homicide, the gendered nature of law and order, the pathologization of women, the * syndromisation" phenomenon and the medicalization of violence, the author offers some insights in thinking legal strategies but more fundamentally in the social debate to continue.

Cet article tente de tracer l'émergence de la reconnaissance sociale et légale de la violence conjugale dans le traitement judiciaire des femmes battues qui ont tué leur conjoint, et déterminer comment l'acceptation récente du "syndrome de la femme battue " (SFB) devant les tribunaux canadiens marque un point tournant dans cette reconnaissance. Dans le but, toujours, de retracer la reconnaissance de ce phénomène, nous allons procéder à un survol général de différents aspects qui nous semblent importants pour offrir une vue d'ensemble.

C'est à la suite d'une décision de la Cour suprême du Canada en $1990^{2}$ que cette question s'est posée avec beaucoup d'acuité. Angélique Lyn Lavallée a été acquittée du meurtre de son conjoint grâce à la preuve d'expert sur la question du «syndrome de la femme battue » (SFB) ${ }^{3}$. Nous utiliserons cette décision comme point central de notre argumentation.

Ainsi, nous nous proposons ici de jeter quelques jalons ou paramètres qui peuvent éclairer notre compréhension de l'homicide conjugal au

1. L'auteure est professeure adjointe au département de criminologie de l'Université d'Ottawa, Ottawa, Ont. KIN 6 N5.

2. R. c. Lavallée [1990] 1 R.C.S. 852.

3. En 1984, la Cour suprême du New Jersey, aux États-Unis, fut l'une des premières cours à accepter la preuve d'expert sur le «syndrome de la femme battue " dans la défense des femmes battues qui tuent leur conjoint (Dobash et Dobash, 1992 : 228). 
féminin ${ }^{4}$. Pour ce faire, cet article comprendra cinq parties : 1) une mise en contexte ; 2) une perspective historique ; 3 ) un bref aperçu de la littérature ; 4) quelques décisions légales; et 5) quelques réflexions et stratégies futures.

Toutes ces parties ont leur importance car elles permettent de mieux comprendre et situer la portée de cette question et le contexte dans lequel elle s'inscrit. Cinq raisons principales motivent le traitement d'ensemble proposé ici. Premièrement, peu d'écrits ont tenté de circonscrire la question dans son ensemble. Deuxièmement, ces diverses parties permettent de cerner le passage de la non-reconnaissance à la reconnaissance de la violence conjugale dans le traitement judiciaire des femmes battues qui ont tué leur conjoint. Troisièmement, elles mettent en évidence, tour à tour, l'absence de la perspective des femmes. Quatrièmement, afin de mieux comprendre les décisions légales (partie 4), il nous est apparu essentiel de situer la problématique de l'homicide conjugal dans son ensemble (partie 1), de présenter un portrait de l'histoire (partie 2) et de la littérature (partie 3) dans le but d'amorcer quelques réflexions et d'envisager certaines stratégies futures (partie 5). Cinquièmement, l'articulation de ces différentes parties permet de mieux comprendre le contexte d'émergence du «syndrome de la femme battue » (SFB) et la façon dont la perspective des femmes a été construite et mise en valeur dans la décision Lavallée en 1990, avec les limites qui y sont inhérentes.

\section{MISE EN CONTEXTE}

Certaines données statistiques sur l'homicide conjugal permettent de mieux situer la problématique. Aux États-Unis, on estime que moins de $1 \%$ des femmes battues tuent leur partenaire. Par ailleurs, $30 \%$ des femmes victimes d'homicide ont été tuées par leur conjoint ou compagnon, et seulement $6 \%$ des victimes de sexe masculin ont été tuées par leur compagne ou conjointe (Boisvert, 1991:192). Au Canada, entre 1961 et 1990, 2129 conjointes ont été tuées par leur conjoint et 782 conjoints ont été tués par leur conjointe (Silverman et Kennedy, 1993 :70). Selon Wilson, Daly et Wright (1993), les femmes mariées ont neuf fois plus de chances d'être tuées par leur mari que par un étranger. Toujours selon ces auteurs, les risques d'assassinat pour les épouses sont beaucoup plus élevés à la suite d'une séparation. Parallèlement, une étude a révélé que de 1974 à 1990, 417 femmes et 141 hommes ont été tués par leur partenaire dans la province

4. Certains auteurs utilisent le terme «uxoricide» pour parler de l'homicide conjugal perpétré par les hommes, car le terme réfère spécifiquement au meurtre de l'épouse par le mari (Wilson, Daly et Wright, 1993), tandis que d'autres parlent de "fémicide conjugal » (Radford et Russell, 1992) ou de "fémicide intime " (Crawford et Gartner, 1992). 
de l'Ontario (Crawford et Gartner, $1992: 34$ ). Enfin, les données du Centre canadien de la statistique juridique indiquent que $14 \%$ des homicides conjugaux commis entre 1974 et 1986 l'ont été par des femmes (Côté, 1991). Ainsi, il ne fait aucun doute que les femmes courent beaucoup plus de risques d'être tuées par un conjoint que l'inverse ${ }^{5}$.

Toutefois, malgré la plus grande fréquence du fémicide conjugal, nous désirons nous pencher, dans cet article, sur l'homicide conjugal féminin pour mettre en relief la construction sociale et pénale du discours sur les femmes qui commettent ces meurtres et le continuum victimisation-criminalisation. Aussi, comme les médias en témoignent, la réaction sociale face aux femmes qui ont tué leur conjoint semble suggérer une plus grande reconnaissance de la violence conjugale dans ces cas ${ }^{6}$.

Au cours des années 1980 , de nombreux changements sont survenus dans différents pays dans le domaine de la reconnaissance légale de la violence conjugale dans les cas d'homicide conjugal féminin. En effet, grâce à ces changements juridiques, la clémence et le pardon ont été octroyés à des femmes dans certains États américains (Noonan, 1993; Hale et Menniti, 1995). La clémence a ainsi été accordée à 26 femmes dans l'Ohio en décembre 1990. Dans le Maryland, en février 1991, 8 femmes qui avaient tué leur partenaire violent ont été libérées ${ }^{7}$. La Grande-Bretagne (O'Donovan, 1991) et l'Australie (Sheehy, Stubbs et Tomlie, 1992; et Tomlie et Stubbs, 1994) sont également parmi les pays qui ont considéré cette preuve d'expert. Au Canada, à la suite de la décision Lavallée et à la

5. Selon certains auteurs, il semble que cette situation ne reflète pas celle de l'Amérique (voir Wilson et Daly, 1992, par exemple). Cette question mérite toutefois plus de recherche, et des auteurs ont remis en question le mythe de la symétrie de la violence conjugale récemment mis de l'avant (Dobash et Dobash, 1992; Wilson et Daly, 1992). Le questionnement poursuivi ici s'inscrit à l'intérieur d'un mouvement plus global de remise en question des paramètres socio-juridiques historiquement établis, tout en s'offrant également comme une étude de cas sur la résistance des femmes face à la violence conjugale.

6. Les titres des journaux témoignent de l'intérêt grandissant au Canada pour cette question: "Battered wives sometimes turn to murder", Calgary Herald, 30 décembre 1984 ; "La Cour suprême acquitte une femme battue qui a tué ", Le Devoir, mai 1990 ; «Légitime défense : Une enquête récente a prétendu que les femmes étaient plus violentes que leur conjoint. Absurde. Et irresponsable », Le Devoir, 25 novembre 1992 ; «Women urge courts to recognize when they are... DRIVEN TO KILL», The Ottawa Citizen, 20 mars 1994; et «Battered woman, 61 gets 3 years probation for stabbing husband », The Toronto Star, 21 septembre 1994.

7. L'acceptation de la preuve d'expert sur le SFB varie selon les États. Par exemple, les tribunaux du Missouri, de la Louisiane et de l'Oregon ont rejeté cette preuve d'expert, tandis que le Maine, l'État de New York, la Californie, l'Onio, la Floride, la Géorgie, I'Illinois, te Kansas, le Kentucky et le New Jersey, notamment, acceptent, à divers degrés, cette preuve d'expert pour établir la légitime défense (Hale et Menneti, $1995: 211$ ). 
demande de la Société Elizabeth Fry, une révision (en bloc) des dossiers des femmes condamnées pour homicide commis dans le contexte d'une relation empreinte de violence est présentement en cours. Mais, comme l'histoire en témoigne, la violence faite aux femmes qui tuaient n'a pas toujours été reconnue dans le traitement judiciaire de ces dossiers.

\section{PERSPECTIVE HISTORIQUE}

Historiquement, le système pénal réagissait autrefois très sévèrement dans les cas des femmes qui se défendaient dans des situations de violence intime, puisque la violence que subissaient les conjointes était en fait tolérée et mêrne acceptée par la société et le système judiciaire. L'adoption de la « règle du pouce » en est un exemple. Comme le souligne la juge Wilson, dans l'arrêt Lavallée, la violence conjugale était acceptée dans le droit :

Loin de les protéger, le droit a dans le passé sanctionné la violence contre les femmes à l'intérieur du mariage en tant qu'aspect du droit de propriété du mari sur sa conjointe et de son «droit» de la châtier. Qu'on se rappelle simplement la loi, en vigueur il y a plusieurs siècles, autorisant un homme à battre sa femme avec un bâton « d'une épaisseur ne dépassant pas celle de son pouce» (la juge Wilson dans R. c. Lavallée, [1990] 1 R.C.S.852: 872).

Qui plus est, selon la loi anglaise, les femmes qui tuaient leur conjoint pouvaient être accusées de "petite trahison», risquant de mener à la peine de mort. Gavigan (1989-1990) a étudié la loi anglaise de "petite trahison» (meurtre d'un mari par sa femme ou d'un maître par son domestique, notamment ${ }^{8}$ ) et constate que de 1351 à 1828 , une femme accusée d'avoir tué son mari pouvait être inculpée de ce crime et non de meurtre prémédité. Or, jusqu'en 1790, cela pouvait entraîner l'exécution publique sur le bûcher. L'histoire de Mme Corriveau en 1763 au Québec illustre bien cette situation. Elle admet avoir tué son mari mais affirme que le meurtre est le résultat de mauvais traitements subis aux mains de la «victime». Lors de son procès, Marie-Josephte Corriveau, veuve Dodier, déclare :

[...] qu'elle a assassiné son mari Louis-Hélène Dodier pendant la nuit alors qu'il dormait dans son lit [...] Elle ajoute que c'est vraiment $d \hat{u}$ en grande partie aux mauvais traitements de son mari si elle est coupable de ce crime (cité dans Collectif Clio, 1992 :136; je souligne).

Comme c'était la tradition au XVIII ${ }^{\mathrm{e}}$ siècle, son corps fut mis en exposition. Elle a été suspendue pendant un mois dans une cage à Lauzon, au Québec (Collectif Clio, 1992:136; Lebel, 1981 :197-201).

8. La question de subordination est centrale, voire essentielle, pour l'existence de cette accusation. Il n'est donc pas surprenant que le meurtre de l'épouse par le mari ne soit pas considéré comme «petite trahison». 
Or, la violence conjugale était complètement ignorée comme circonstance atténuante dans ce type de procès, et les femmes qui résistaient à l'ordre masculin étaient punies très sévèrement afin de dissuader les autres femmes d'en faire autant ${ }^{9}$. Comme nous allons le montrer très brièvement maintenant, l'histoire du Canada est ponctuée d'exemples similaires.

Le dépouillement d'archives canadiennes et plus particulièrement le répertoire des dossiers individuels conservés dans les archives du ministre de la Justice (RG 13) sur les condamné(e)s à la peine de mort au Canada pour la période 1867-1976 (Gadoury et Levasseur, 1994) nous a permis d'analyser les dossiers de femmes ayant tué leur conjoint ${ }^{10}$. Pour des raisons méthodologiques et de découpage historique, nous avons procédé, dans un premier temps, à l'examen des dossiers des femmes au Canada anglais pour, ensuite, nous concentrer dans un deuxième temps sur ceux des femmes au Canada français. Les commentaires qui suivent portent uniquement sur les dossiers de femmes au Canada anglais.

Ces documents sont une source très riche d'informations quant à la perception de la nature et du rôle de la femme et de la mère, ainsi qu'à la position des femmes dans le mariage et dans la société. Ces perceptions sont fournies à travers les yeux des agents du droit (policiers, avocats et juges), de la médecine (médecins et psychiatres), de la famille (enfants, sœurs et frères) et de la société (voisins, auteurs de pétitions et articles de journaux).

Dans bon nombre de dossiers, on note la présence de la violence conjugale. Même si celle-ci ne semble pas jouer un rôle déterminant quant à la reconnaissance de la culpabilité de la femme, on peut se demander si elle n'en joue pas un au niveau du prononcé de la sentence. En effet, dans presque tous les cas, la peine de mort sera commuée en une très longue incarcération. Dans la plupart des cas analysés, même si la violence conjugale a été établie par les témoignages (des médecins, policiers, enfants, sœurs et/ou voisins), elle tiendra un rôle secondaire car le rôle attendu de l'épouse et de la mère prendra l'avant-scène.

En effet, dans les 18 dossiers de femmes condamnées à la peine de mort, trois ont été exécutées en Ontario: Phoebe Campbell en 1871 à London, Elizabeth Workman en 1873 à Sarnia, et Elizabeth Tilford en 1935

9. Une pièce de thêâtre sur la vie et la mort de Marie-Josephte Corriveau a été présentée aux Beaux Dimanches de Radio-Canada le 15 janvier 1995. La violence vécue par Mme Corriveau fut explicitée dans cette pièce. Le lieutenant-colonel Morris justifia la sentence exemplaire en expliquant: "L'exemple doit être assez fort pour prévenir les crimes pendant les prochaines années. $S$ 'il fallait que les épouses mécontentes de leur son assassinent leur mari, il n'y aurait plus d'hommes dans la colonie [...]» (Lebel, $1981 ; 180)$.

10. Pour une discussion plus détaillée de ces résultats, voir Frigon (1995). 
à Woodstock. En 1911, à Sault Sainte Marie, en Ontario également, Angelina Napolitano fut condamnée à la peine de mort mais sa sentence fut commuée en raison de sa grossesse. Les autres sentences ont été commuées en peines d'incarcération variant de 7 ans (pour des raisons de santé) jusqu'à 17 ans. Deux femmes ont finalement été acquittées et une autre a été acquittée pour aliénation mentale. Dans 10 des 18 cas, la violence conjugale était explicite et documentée mais n'a pas eu de pertinence légale auprès des juges.

Comme en témoignent ces procès, la violence conjugale dans les cas de femmes qui tuent leur conjoint tient une place importante dans le déroulement des événements, mais sa portée juridique est très limitée Cette idée a aussi été véhiculée dans les écrits de l'époque.

\section{LA LITTÉRATURE : QUELQUES JALONS}

Il est important de souligner que l'intérêt pour le sujet des femmes qui ont tué leur conjoint n'est pas nouveau. Dès 1895, Lombroso et Ferrero, considérés comme les "pères fondateurs" de la criminologie positiviste traditionnelle, soulignaient, quoique seulement au passage, que l'homicide conjugal féminin pouvait surgir d'une réaction et d'une révolte contre les abus et l'humiliation vécus par ces femmes aux mains de leur conjoint. Ces auteurs fournissent quelques études de cas qui illustrent cette problématique. Рar exemple :

Mme Daru, très honnête fermme, était continuellement battue, ainsi que ses enfants, par son mari ivrogne. Menacée un jour d'un couteau, elle s'enfuit avec eux de la maison: elle ne rentra que lorsqu'elle le crut endormi et le tua de coups de couteau (Lombroso et Ferrero, 1895: 411).

Cette Mme R. B..., qui avait été mariée par sa mère à un de ses amants, vieux et brutal, qui la battait parce qu'elle se refusait à lui, et lui faisait endurer le froid et la faim, lasse de souffrir, mêla un jour du sulfate de cuivre à la polenta et en fit manger à son mari [...] (ibid:414).

Nous devons attendre, néanmoins, les années 1930 pour que des études plus étoffées sur le lien entre la violence conjugale et les femmes qui tuent fassent leur apparition. Un nombre important d'auteurs se sont penchés sur cette question (Brearley, 1932; Morton, 1934; Matheson, 1939; Von Hentig, 1940, 1948 ; et Harlan, 1950). Pour sa part, Harlan soutient que «dans un nombre considérable de meurtres... le mari ou l'amant est tué lorsque la femme se défend contre ses coups " (1950:750; ma traduction). L'étude la plus influente des années 1950 est celle de Wolfgang (1956). Dans son échantillon de 100 cas d'homicide conjugal, il démontre que $41 \%$ des femmes victimes ont été tuées par leur conjoint, contre seulement $11 \%$ des hommes qui ont été tués par leur conjointe. Toujours selon cet auteur, les 
maris auraient «précipité » leur mort en se montrant violents à l'égard de leur conjointe.

Dans les années 1960, comme le souligne Rasche (1990), des études de deux types sont apparues: 1) des recherches empiriques et statistiques; et 2) des études psychologiques et psychiatriques. Si les premières ont montré que l'homicide conjugal était majoritairement le fait des hommes, les secondes ont fait ressortir les dimensions psychologiques et psychiatriques dans la compréhension de ce phénomène (Cole, Fisher et Cole, 1968). Plus tard vont apparaître des études socio-juridiques et féministes qui vont mettre l'accent non pas seulement sur le caractère sexué $d u$ délit mais plutôt sur le caractère sexué du droit pénal ${ }^{11}$.

La littérature de la fin des années 1980 et du début des années 1990 rompt avec les premières études pour mettre l'accent sur la question de la légitime défense, de la preuve d'expert du «syndrome de la femme battue », en insistant sur la perspective des femmes ${ }^{12}$. Dans ces études, la construction sociale et pénale de ces défenses sera abordée pour exposer leur caractère normatif masculin. En dévoilant cet aspect, les contributions ont ainsi déploré l'absence de la perspective des femmes. Une attention particulière devrait être accordée à cette période, puisqu'il y a, ici, rupture épistémologique importante : les études précédentes avaient adopté la «perspective des hommes » sur les défenses légales comme la provocation et la légitime défense, alors que les recherches des années 1980 problématisent cette perspective en mettant l'accent sur le caractère "sexué » du droit pénal dans ce domaine et sur l'importance et la légitimité de la perspective (ou des perspectives) des femmes. Cette rupture est illustrée par la décision Lavallée.

\section{L'AFFAIRE LAVALLÉE (1990) CONTEXTUALISÉE ET REVISITÉE 13}

Comme nous pouvons le constater, au Canada, un arrêt a fondamentalement transformé la défense des femmes qui ont tué leur conjoint violent grâce à la preuve d'expert sur le SFB. La construction du SFB dans les décisions légales est basée sur des prémisses établies par la psychologue clinicienne américaine Lenore Walker $(1979,1984)$. Le «syndrome de la femme battue " est répertorié dans le DSM-1V, registre américain sur les maladies mentales, sous la rubrique «État de stress post-traumatique ». II

11. En fait, c'est le caractère sexué du droit pénal qui est en procès dans la décision Lavallée et qui est dénoncé par la juge Bertha Wilson, notamment.

12. Un nombre considérable de travaux de langue anglaise sont parus sur la question du «syndrome de la femme battue » et sur la décision Lavallée, tandis que très peu d'études sont parues en langue française. Notre bibliographie en témoigne.

13. Étant donné les nombreuses études juridiques très intéressantes portant sur cette décision, l'examen de ses aspects juridiques en sera sommaire. 
est fait référence à un état " pathologique » de dépendance et d'impuissance qui semble empêcher la personne de fonctionner «normalement ». L'extrait qui suit décrit l'état mental dans lequel Angélique Lyn Lavallée se trouvait au moment de l'attaque, et nous donne une idée des faits qui sont importants, voire décisifs, dans la construction du SFB, tels que le sentiment d'être piégée, la violence répétée et la peur légitime de la mort :

[...] j'ai couru dans la maison quand Kevin m'a poussée. J'avais peur, j'avais vraiment peur. J'ai fermé la porte à clef [...]. J'avais tellement peur [...]. Je me suis rendu compte ensuite qu'il montait me chercher. Il est entré dans ma chambre à coucher et a dit : «Salope, où es-tu? » [...] O.K. puis il s'est retourné et m'a vue dans le placard. Il voulait que je sorte, mais moi je ne voulais pas parce que j'avais peur. J'avais tellement peur [...]. Il m'a saisie par le bras juste là. J'ai aussi un bleu au visage là où il m'a giflée. J'avais peur. Je ne pensais plus qu'à toutes les autres fois qu'il m'avait battue; j'avais peur; je tremblais comme d'habitude. Il a dit: «Attends que tout le monde soit parti, tu auras de mes nouvelles ", et puis il a dit quelque chose du genre de : «Ou tu me tues, ou moi je t'aurai »[...] [R. c. Lavallée, [1990], à la page 856$]$.

Le syndrome de la femme battue ne constitue pas, en soi, une défense, comme le sont la provocation ou la légitime défense, mais il remet en question notre conception de légitime défense puisque avant 1990, au Canada, la question de déterminer si quelqu'un était raisonnable était jugée selon le standard de 1 '« homme raisonnable ${ }^{14} »$. Alors, même si le Code criminel canadien admet que, légalement, quelqu'un peut causer la mort dans le but de se défendre (article 34.2), cette défense semble plutôt être efficace pour les hommes qui tuent. Par exemple, la légitime défense a été établie pour une situation qui se pose entre hommes qui ne se connaissent pas, de poids, taille et force similaires (bar room brawl) et non dans le cas de la violence entre un homme et une femme ou dans un cas de violence conjugale. Les abus de longue durée ne sont pas considérés. Ces critères jurisprudentiels n'incluent pas la réalité des femmes, si bien qu'elles se trouvaient à l'extérieur de cette logique pénale.

Cela est aussi lié à une autre difficulté, celle du critère de l'attaque imminente. L'application controversée de ce critère en 1983 en NouvelleÉcosse dans le cas $R$. v. Whynot mérite d'être relevée: Jane Stafford fait feu sur son mari pendant qu'il dormait. Ce soir-là, il avait menacé de tuer son fils. Il avait violenté Jane Stafford et les autres membres de la famille, mais l'imminence de l'attaque et la légitime défense ont été considérées comme non fondées. Elle fut accusée de meurtre. Par ailleurs, un examen critique

14. La construction légale de l'homme raisonnable a été remise en question fort éloquemment, notamment, par Allen (1987). 
de ces principes existe dans Lavallée. Sur la question de l'imminence de l'attaque, la juge Wilson écrit dans la décision Lavallée :

L'exigence, posée dans l'arrêt Whynot, qu'une femme battue attende que l'agression soit «en cours » pour que ses appréhensions soient reconnues comme juridiquement valables reviendrait, pour reprendre la formule d'un tribunal américain, à la condamner au [traduction] «meurtre à tempérament » [...] (R. c. Lavallée, [1990], p. 883).

Ainsi, dans le cas de Mme Lavallée, on a établi que les perceptions et les actions de l'accusée étaient «raisonnables", et cela est un acquis fondamental. En effet, son acte sera reconnu comme légitime et non pas simplement excuséls.

Maintenant, le SFB constitue une certaine preuve amenée par un expert quant au phénomène social et psychologique qu'est la violence conjugale. Dans le cas d'Angélique Lyn Lavallée, on a démontré que la victime, Rust, était un homme violent envers ladite accusée. On peut lire dans le jugement :

Les rapports entre l'appelante et Rust étaient explosifs, ponctués de fréquentes altercations et de violence. Apparemment, ils se disputaient deux ou trois jours de suite ou plusieurs fois par semaine. D'après une preuve abondante produite au procès, l'appelante a souvent été brutalisée par Rust. Entre 1983 et 1986, elle est allée plusieurs fois à l'hôpital faire soigner des blessures, dont des meurtrissures graves, une fracture du nez, des contusions multiples et un æil poché (R. c. Lavallée, [1990], p. 857).

Or, l'expert dans cette affaire, le $\mathrm{D}^{\mathrm{r}}$ Shane, psychiatre, était en accord avec la théorie de Walker au sujet du caractère cyclique de la violence. Walker décrit ce cycle pour la première fois dans son livre The Battered Women (1979). Plus tard, dans The Battered Woman Syndrome (1984), elle le résume ainsi :

Suivant cette théorie de la réduction de tension, le cycle de violence répétée comporte trois phases distinctes: (1) l'accroissement de la tension,

15. Rappelons simplement ici, pour les besoins de notre discussion, la distinction entre justification et excuse en matière criminelle et son importance pour les femmes battues qui tuent. Sur ce sujet, Boisvert (1991) écrit : « Alors que la justification a pour effet de nier le caractère mauvais de l'acte, en reconnaissant qu'il a été légitimement commis, l'excuse, tout en affirmant le caractère mauvais de la conduite, reconnaît la présence d'un motif qui justifie la compassion pour son auteur " (194). En conséquence, comme le souligne toujours Bojsvert, la littérature féministe « insiste particulièrement sur l'importance de l'accès pour les femmes battues à la légitime défense par opposition à la provocation ou aux moyens de défense mettant en cause un état mental déficient ou altêré, telles l'aliénation mentale ou la responsabilité atténuée ". 
(2) l'incident de violence grave et (3) la contrition assortie de manifestations d'amour (cité dans R. c. Lavallée, [1990], p. 879).

Étant donné le contexte relationnel dans lequel cette violence se situe, il faut la comprendre en prenant en considération l'aspect d'intimité et ses effets cumulatifs qui donneront naissance, selon le $D^{r}$ Shane, à «des sentiments croissants de terreur» (ibid: 880 ) chez l'appelante. Par conséquent, un "degré de prévisibilité qu'on ne trouve pas dans un incident isolé de violence entre deux personnes qui ne se connaissent pas" (ibid: 882) permet à la partenaire violentée de prédire une violence "inhabituelle » qui peut mettre sa vie en danger. En effet: "Rust lui a en fait remis une carabine en la prévenant que si elle ne le tuait pas c'était lui qui la tuerait» (ibid: $882^{16}$ ).

Or, comme le souligne Boisvert (1991), «la décision ne crée pas de nouveau moyen de défense pour les femmes battues, mais reconnaît plutôt qu'il est normal de juger une accusée en tenant compte de la perspective des femmes» (194). Qui plus est, en citant une décision américaine (State v. Wanrow, 1977), la juge Wilson écrit que :

L'intimée avait droit à ce que le jury examine ses actes à la lumière de ses propres perceptions de la situation, notamment celles résultant de notre "longue et regrettable tradition de discrimination fondée sur le sexe » (...) Sinon on se retrouvera à refuser à l'intéressée le droit d'être jugée selon les mêmes règles que celles qui s'appliquent aux défenseurs de sexe masculin (ibid: 875).

Ainsi, comme nous l'avons déjà souligné, on a établi que les perceptions et les actions de l'accusée étaient "raisonnables», ce qui constitue un changement paradigmatique dans la construction de la «raison" de la femme. Cette «raison" est toutefois atteinte d'un syndrome, le «syndrome de la femme battue». Maintenant, le SFB constitue une certaine preuve amenée par un expert quant à la violence conjugale et permet d'établir deux éléments : 1) pourquoi elle n'était pas partie et, donc, d'établir son incapacité liée au SFB; et 2) de démontrer le caractère cyclique de la violence.

\subsection{Les conséquences de la décision Lavallée}

Même si cette décision devrait être accueillie comme une victoire féministe et une reconnaissance légale de la violence faite aux femmes, il

16. Cette situation a été aussi retrouvée dans l'étude de Walker (1984) au sujet de 50 femmes battues qui avaient tué leur partenaire: "Dans la plupart des cas, les femmes ont tué l'homme avec une arme à feu, le plus souvent une des armes appartenant à celui-ci. Un grande nombre de ces hommes ont même défié la femme ou lui ont ordonné de tirer la première, disant que sinon ils se serviraient eux-mêmes de l'arme pour la tuer» (à la page 40 cité dans ibid p. 882). 
semble, néanmoins, y avoir quelques risques. Afin de préserver les acquis de cette décision déterminante, nous allons faire ressortir, dans la prochaine section, les problèmes et dérapages possibles d'une lecture moins généreuse de Lavallée. Il semblerait que la défense du SFB ait été évoquée dans différentes décisions depuis. Nous disposons de recherches préliminaires sur le sujet. Par exemple, Sheehy $(1993,1995)$ a pu répertorier une dizaine de cas depuis Lavallée, de 1990 à 1994, où le SFB a été parfois utilisé sans succès. Elle fait état de quelques décisions dans lesquelles le SFB n'a pas été retenu pour établir la légitime défense et donner lieu à l'acquittement ${ }^{17}$. À ce sujet, Sheehy écrit :

Il ressort de l'analyse qu'aucune des femmes qui s'étaient appuyées sur l'arrêt Lavallée n'a été reconnue non coupable d'homicide, et que l'arrêt n'a pas procuré les acquittements qu'il aurait dû. Dans le cas d'au moins 10 femmes, le SFB a plutôt servi à atténuer les peines imposées (1995: 8).

[...] il appert des affaires où des femmes ayant blessé ou tué des hommes violents ont présenté une preuve de SFB dans le but d'atténuer la sanction, que les accusées disposaient en fait d'éléments solides de légitime défense et qu'il aurait été approprié de les acquitter (ibid: 12).

Un examen préliminaire de ces décisions nous permet de voir trois difficultés potentielles se dessiner au sujet de l'utilisation du SFB ${ }^{18}$. Premièrement, il semble que les critères soient trop exigeants sur la définition de la "parfaite victime». Deuxièmement, il y a des stéréotypes racistes dans la définition même du SFB. Ainsi, dans les cas de Howard, Catholique et Eyapaise, le discours semble traversé, selon Sheehy $(1993,1995)$, par des stéréotypes racistes dans la construction de leur féminité autochtone. Dans le cas des femmes blanches, la violence peut être perçue comme une réponse irrationnelle et pathologique en raison du SFB, et donc, celui-ci peut traduire cette perception. Pour leur part, les femmes autochtones ne sont pas perçues comme des «victimes passives» mais comme «naturellement» plus violentes. Le SFB ne pourra être plaidé. Troisièmement, il risque d'y avoir un glissement en faveur du discours psychiatrique dans la compréhension des actes de la femme. Cette troisième difficulté est fort importante puisque dans Lavallée, l'action de l'accusée est perçue comme légitime, alors qu'il semble que les interprétations subséquentes

17. R. c. Dunlap (1991), 101 N.S.R. (2d) 263 (S.Ct. App.Div.); R. c. Whitten (1992), 110 N.S.R. (2d) 149 (S.Ct. T.Div.); R. c. Howard (1992), 8 B.C.A.C. 241 (C.A); R. v. Catholique (1990) N.W.T.J. No 164 (N.W.T. Sup. Ct.); R. C. Bennett [no 1] (1993) (Ont. Prov.Ct.); R. c. Tran (1991) O.J. No 2052 (Ct. J. Gen.Div.); et $R$. c. Eyapaise (1993) 20 C.R. (4th) 246 (Alta. Q.B.).

18. Sheehy (1995) suggère néanmoins, avec une certaine réserve, que les craintes exprimées par des féministes sur l'usage très restreint de Lavallée et de la preuve d'expert sur le SFB ne semblent pas se réaliser. 
risquent de privilégier une explication du type pathologique pour excuser les actions des accusées. Il semble donc important d'examiner pourquoi et comment certaines femmes qui réagissent à la violence par l'agression ne peuvent être reconnues comme «femme battue " et, donc, ne peuvent bénéficier de la preuve d'expert du SFB. Par exemple, il semble que si elles ne remplissent pas les critères de "pathologie" liés au syndrome, elles ne sont pas perçues comme sans pouvoir; ce qui signifie, selon Noonan (1993), que cela nie leur autonomie et leur libre choix ("agency»). Donc, si les comportements des femmes apparaissent comme rationnels et raisonnables, elles seront passibles de sanctions pénales. Plus généralement encore, les limites liées au SFB semblent inhérentes à la nature même du système pénal, comme nous l'avons sommairement souligné précédemment. Mais fondamentalement, le SFB pourrait jouer un rôle déterminant pour établir la légitime défense, et c'est cela qui devrait être remis en question dans une réforme du code criminel (Côté, 1995; Sheehy, 1995).

Or, cela nous amène à problématiser l'utilisation du SFB : s'offre-t-il comme une stratégie de résistance ou comme une stratégie de disqualification pour les femmes? D'une certaine manière, nous pouvons dire que l'admissibilité du SFB au Canada permet de valider les expériences des femmes - expériences jusqu'alors ignorées et évacuées. Dans le cas de Lavallée, par exemple, nous voyons que l'histoire de violence a été prise en compte comme un facteur déterminant dans sa réaction. L'acquittement de Mme Côté au Québec, en juillet 1995, en est aussi un exemple ${ }^{19}$. Victoire certaine de ce point de vue. Par ailleurs, l'expérience des femmes est traduite par la voix d'un expert. Or, a-t-on a écouté la voix des femmes? Dans les cas de Lavallée et de Côté, encore une fois, on peut réinterpréter la stratégie de survie qu'elles ont utilisée comme un état pathologique par la voie/voix du SFB. La reconnaissance légale du SFB est-elle done moins une reconnaissance de la perspective des femmes qu'une reconnaissance du pouvoir des «psy» pour traduire l'expérience des femmes (Smart, 1989; Comack, 1993)?

\section{RÉFLEXIONS ET STRATÉGIES FUTURES}

Au début des années 1980, aussi bien au Canada qu'ailleurs, sont apparues des études sur la question des femmes ayant tué leur conjoint violent et la pertinence légale du SFB dans une perspective féministe et critique (Walker, 1984; Sheehy, 1987, 1993, 1995; Comack, 1993; Noonan, 1993, entre autres ${ }^{20}$ ). La plupart de ces études font état du caractère masculin du droit et de l'impératif de tenir compte de la perspective des femmes

19. C.A.Q., Dossier no 700-01-004987-924.

20. Malgré l'importance de ces contributions, il est important de noter qu'elles sont très souvent ignorées, comme le souligne Sheehy (1995). 
et donc rompent avec une tradition empirique vouée à mettre en relief la participation des femmes dans l'homicide conjugal ${ }^{21}$.

À la suite de ces travaux de nature presque exclusivement juridique, il est important d'examiner la question dans une perspective plus globale. Cette approche permet d'apporter un nouvel éclairage et de contribuer, de façon originale, aux débats actuels dans le domaine. Nous pouvons noter quelques aspects qui posent certaines difficultés et qui sont rarement problématisés en profondeur dans les études dont nous disposons actuellement. Nous devons examiner, par exemple, la question du "syndrome " et de ses conséquences. Cette «syndromisation » risque de médicaliser et de pathologiser les femmes victimes de violence conjugale, même s'il a permis, dans un premier temps, de faire reconnaître leurs expériences. Aussi, elle s'apparente à l'utilisation de trois autres syndromes en droit : le "syndrome prémenstruel » (Kendall, 1992), le «syndrome de la femme violée » (Delorey, 1989) et le "syndrome de la fausse mémoire", qui ont aussi eu des conséquences néfastes pour la représentation des femmes. Deuxièmement, le fait que le SFB soit répertorié dans le DSM-1V, comme les autres syndromes mentionnés ci-dessus, peut renforcer l'idée de pathologie. Troisièmement, le SFB comme défense utilisée devant les tribunaux peut avoir des conséquences au niveau de la construction sociale et pénale de la féminité; par exemple, dans la construction de la raison des femmes. Comme le suggère Ann Jones, auteure de When Women Kill:

The courts have never been able to deal with women who kill particularly women who kill men [...] so the courts have bent over backwards to try and find ways to explain that in terms of the particular psychological aberration of the woman. In the 19th century [...] the courts would acknowledge that indeed she must have been insane at this moment and perhaps she would be acquitted of the crime. That's the pattern that has continued right up until the present. The courts still entertain pleas of insanity from women only nowadays we do it in a more sophisticated way so that in England the PMS defense, the premenstrual syndrome defense was popular to explain why a woman committed the irrational act of killing the husband. In the U.S. and now in Canada we have tumed to the Battered Woman Syndrome (When Women Kill, Office national du film du Canada, 1994).

Il semble, en effet, que la raison des femmes soit souvent perçue comme posant problème. Quatrièmement, le fait d'utiliser le SFB assure la médicalisation et même la pathologisation du corps des femmes et de leurs actions

21. Le SFB a aussi été utilisé dans d'autres situations telles que le vol qualifié, l'homicide involontaire d'un partenaire (soi-disant non violent), le trafic de stupéfiants (voir Sheehy, 1995: 9) et, plus récemment, en 1995, dans le cas d'une femme de Sudbury accusée de fraude de prestations de l'aide sociale. 
et expériences, et positionne le corps des femmes comme site de contrôle en justifiant l'intervention psychiatrique et la médication. Et, finalement, une utilisation très stricte du SFB peut occulter l'analyse plus globale des rapports de sexe.

Sheehy, Stubbs et Tolmie (1992) recommandent toutefois de sauvegarder cette preuve d'expert en faisant deux choses: en abandonnant le terme de SFB et en le démédicalisant. Ces auteurs suggèrent, par exemple, que les experts pouvant témoigner dans ces causes ne soient pas uniquement des psychiatres mais que des intervenant(e)s auvrant dans des centres d'aide pour femmes violentées puissent aussi contribuer à cette question. Plus récemment, Sheehy (1995) posait des jalons juridiques pour une défense de légitime défense pour les femmes ${ }^{22}$. De plus, comme stratégie légale, la révision des dossiers des femmes condamnées pour homicide commis dans le contexte d'une relation empreinte de violence en cours depuis l'automne 1995 a pour objectif d'analyser les dossiers de femmes qui ont tué dans une perspective globale, et de mettre en relief la violence conjugale et la violence faite aux femmes ${ }^{23}$.

Comme nous pouvons le constater, l'utilisation du syndrome de la fermme battue est un élément très important dans la défense des femmes en droit, mais il pose aussi certaines difficultés quant à la représentation de la féminité. Comme l'histoire nous l'enseigne, la reconnaissance de la violence conjugale est un acquis fort récent, et le SFB s'offre comme une solution de remplacement aux défenses traditionnelles jusqu'ici inadéquates pour les femmes qui tuent un conjoint violent. Mais la voix des femmes est-elle vraiment entendue ? Ou est-ce que, au contraire, la voix des "psy» n'est pas validée aux dépens des femmes? Or, pour que le SFB devienne une stratégie de résistance pour les femmes, il faudra sauvegarder les acquis de Lavallée et deux démarches légales spécifiques sont présentement en cours pour atteindre cet objectif : 1) un examen du Code criminel canadien pour rendre compte des perspectives des femmes, et 2) la révision des dossiers des femmes condamnées pour homicide dans le contexte d'une rclation empreinte de violence. D'autres stratégies théoriques et pratiques

22. Sheehy réitère l'idée qu'elle avait avancée en 1987, celle de la défense d'autopréservation ou de préservation des autres. Cette défense offre plusieurs avantages et devrait être prise en considération très sérieusement.

23. Il faut garder en tête ici la question du continuum victimisation-criminalisation. En effet, le groupe d'études La Création de choix (1990) avait prouvé, documents à l'appui, qu'une très grande majorité des femmes condamnées à une peine fédérale (à des peines d'emprisonnement de deux ans et plus) avaient un passé de victimisation. On parlait de 60 à $90 \%$ des cas. Plus récemment, le rapport sur la condition des femmes dans le système correctionnel, Voix des femmes. Choix des femmes (1995), sur les femmes condamnées à une peine provinciale (à moins de deux ans d'emprisonnement), se penche aussi sur cet aspect. Cette question mérite une attention particulière. 
doivent aussi se greffer aux stratégies légales pour tenter de cerner la complexité de la question que pose la problématique des femmes battues qui tuent et aussi l'utilisation du SFB. Ce texte, nous l'espérons, aura contribué à ce projet.

\section{BIBLIOGRAPHIE}

ALLEN, H. (1987), Justice Unbalanced - Gender, Psychiatry and Judicial Decisions, Milton Keynes, Open University Press.

AMERICAN PSYCHIATRIC ASSOCIATION (1994), Diagnostic and Statistical Manual of Mental Disorders, (4th ed. rev.), Washington, D.C., American Psychiatric Press.

BOISVERT, A.-M. (1991), "Légitime Défense" et le «syndrome de la femme battue ": R. c. Lavallée, Revue de droit de McGill, vol. 36, pp. 191-215.

BOYLE, C. et al, Un examen féministe du droit criminel, Canada : ministère des Approvisionnements et Services Canada.

BREARLEY, H. (1932), Homicide in the United States, Chapel Hill, University of North Carolina Press.

COLE, K., FISHER, G. et COLE, S. (1968), «Women Who Kill », Archives of General Psychiatry, vol. 19, pp. 1-8.

COLLECTIF CLIO (1992), L'histoire des femmes au Québec depuis quatre siècles, Montreal, Le Jour.

COMACK, E. (1993), Feminist Engagement with the Law : The Legal Recognition of the "Battered Woman Syndrome", Ottawa, Canadian Institute for the Advancement of Women Papers, $n^{\circ} 31$.

CÔTÉ, A. (1991), La Rage au Caur : rapport de recherche sur le traitement judiciaire de l'homicide conjugal au Québec, Regroupement des femmes de la Côte-Nord.

CôTÉ, A. (1994), La défense de provocation et le fémicide conjugal, mémoire de maitrise, Faculté de droit, Université de Montréal.

CôTÉ, A. (1995), La violence contre les femmes et la réforme du droit criminel: Recommandations en vue d'une réforme égalitaire du droit criminel, Ottawa, Action ontarienne contre la violence faite aux femmes.

CRAWFORD, M. et GARTNER, R. (1992), Woman Killing : Intimate Femicide in Ontario, 1974-1990, Toronto, Women We Honour Action Committee.

CUSSON, M. et BOISVERT, R. (1994), «L'homicide conjugal à Montréal, ses raisons, ses conditions et son déroulement », Criminologie, vol. XXVll, $n^{\circ} 2$, pp. 165-184.

DELOREY, A.-M. (1989-1990), «Rape Trauma Syndrome : An Evidentiary Tool ", The Canadian Journal of Women and the Law/Revue femmes et droit, vol. 3 , pp. 531-551.

DOBASH, R.E. et DOBASH R.P. (1992), Women, Violence and Social Change, London, Routledge.

FRIGON, S. (1995), «When Women Kill Violent Husbands in Canada, 1871-1946: Drama, Disqualification of Women's Voices, Resistance and Male Tyranny ", 
Conférence présentée à la British Criminology Conference, à l'Université de Loughborough en Angleterre du 18 au 21 juillet 1995.

GADOURY, L. et LECHASSEUR, A. (1994), Les condamnésles à la peine de mort au Canada, 1867-1976: un réperioire des dossiers individuels conservés dans les archives du ministre de la Justice ( $R G$ 13), Ottawa, Archives nationales du Canada.

GAVIGAN, S. (1989-1990), "Petit Treason in Eighteenth Century England: Women's Inequality Before the Law ", The Canadian Journal of Women and the Law/Revue femmes et droit, vol. 3, $\mathrm{n}^{\circ} 2$, pp. 335-374.

HALE, D. et MENNET], D. (1995), "The Battered Woman Syndrome as Legal Defense : Status in the Courtroom», Women, Law, \& Social Control, Merlo, A. et Pollock, J. (éds), Toronto, Allyn and Bacon, pp. 203-218.

HARLAN, H. (1950), "Five Hundred Homicides ", Journal of Criminal Law, Criminology and Police Science, vol. 40, pp. 736-752.

KENDALL, K. (1992), "Sexual Difference and the Law : Premenstrual Syndrome as Legal Defense ", The Anatomy of Gender, Currie, D. et Raoul V. (eds.), Ottawa, Carleton University Press, pp. 130-146.

LEBEL, A. (1981), La Corriveau, Montmagny, Éditions Libre Expression.

LOMBROSO, C. et FERRERO, G. $\left(1991 ; 1^{\text {re }}\right.$ éd. : 1895), La femme criminelle et la prostituée, Grenoble, Éditions Jérôme Millon.

MATHESON, J. C. M. (1939), "Alcohol and Female Homicides", The British Journal of Inebriety, vol. 37, pp. 87-90.

MORTON, J. (1934), "Female Homicides», Journal of Mental Science, vol. 80, pp. 64-74,

NOONAN, S. (1993), «Strategies of Survival : Moving Beyond the Battered Woman Syndrome ", In Conflict with the Law : Women and the Canadian Justice System, Adelberg, E. et Currie, C. (éds), Vancouver, Press Gang Publishers, pp. 247.27.

O'DONOVAN, K. (1991), «Defences for Battered Women Who Kill », Journal of Law and Society, 18.

RADFORD, J. et RUSSELL, D. (1992), Femicide: The Politics of Woman Killing, Buckingham, Open University Press.

RASCHE, C. (1990), «Early Models for Contemporary Thought on Domestic Violence and Women Who Kill Their Mates: A Review of the Literature from 1895 to $1970 »$, Women \& Criminal Justice, vol. 1, $\mathrm{n}^{\circ} 2$, pp. 1-43.

R. c. Côté, C.A.Q., Dossier n 700-01-004987-924.

R. c. Lavallée (1990), 76 C.R. (3rd) 329 (S.C.C.).

R. c. Dunlap (1991), 101 N.S.R. (2d) 263 (S.Ct. App.Div.).

R. c. Whitten (1992), 110 N.S.R. (2d) 149 (S.Ct. T.Div.).

R. c. Howard (1992), 8 B.C.A.C. 241 (C.A).

R. v. Catholique (1990) N.W.T.J. No 164 (N.W.T. Sup. Ct.).

R. c. Bennett [ $\left.\mathrm{n}^{0} 1\right]$ (1993) (Ont. Prov.Ct.).

R. c. Tran (1991) O.J. $\mathrm{N}^{\circ} 2052$ (Ct. J. Gen.Div.).

R. c. Eyapaise (1993) 20 C.R. (4th) 246 (Alta. Q.B.). 
SER VICES CORRECTIONNELS DU CANADA (1990), La Création de choix : Rapport du groupe d'étude sur les femmes purgeant une peine fédérale. Ottawa, ministère des Approvisionnements et Services.

SERVICES CORRECTIONNELS DU CANADA (1995), Voix de femmes, Choix de femmes: Rapport du groupe de travail sur la condition des femmes dans le système correctionnel, Ottawa, ministère des Approvisionnements et Services.

SHEEHY, E. (1987), Personal Autonomy and the Criminal Law : Emerging Issues for Women. Ottawa, Canadian Advisory Council on the Status of Women.

SHEEHY, E., STUBBS, J. et TOMLJE, J. (1992), «Defending Battered Women on Trial : The Battered Woman Syndrome and its Limitations ", Criminal Law Journal, vol. 16, pp. 369-394.

SHEEHY, E. (1993), «Developments in Canadian Law After R. v. Lavallée ", document non publié.

SHEEHY, E. (1995), À quoi ressemblerait le droit de la légitime défense pour les femmes? Ottawa, Condition féminine Canada.

SILVERMAN, R. et KENNEDY, L. (1993), Deadly Deeds : Murder in Canada, Scarborough, Nelson Canada.

SMART, C. (1989), Feminism and the Power of Law, London, Routledge.

TOMLIE, J. et STUBBS, J. (1994), «Battered Woman Syndrome in Australia: A Challenge to Gender Bias in Law?», Women, Male Violence and the Law, J. Stubbs (éd.), Sydney, Institute of Criminology.

VON HENTIG, H. (1940), «Remarks on the Interaction of Perpetrator and Victim ", Journal of Criminal Law and Criminology, vol. 31, pp. 303-309.

VON HENTIG, H. (1948), The Criminal and His Victim, New York, Schoken Books.

WALKER, L. (1979), The Battered Women, New York, Harper \& Row.

WALKER, L. (1984), The Battered Woman Syndrome, New York, Harper Colophon Books.

WILSON, M. et DALY, M. (1992), "Who Kills Whom in Spouse Killings? On the Exceptional Sex Ratio of Spousal Homicides in the United States », Criminology, 30, pp. 301-327.

WILSON, M., DALY, M. et WRiGHT, C. (1993), "Uxoricide in Canada : Demographic Risk Patterns », Canadian Journal of Criminology/Revue canadienne de criminologie, pp. 263-291.

WOLFGANG, M. (1956), «Husband-Wife Homicides», Journal of Social Therapy, vol. 2, pp. 263-271. 